\title{
Long non-coding RNA ANRIL is upregulated in hepatocellular carcinoma and regulates cell proliferation by epigenetic silencing of KLF2
}

Ming-de Huang ${ }^{1 \dagger}$, Wen-ming Chen ${ }^{2 \dagger}$, Fu-zhen Qi ${ }^{3 \dagger}$, Rui Xia ${ }^{4}$, Ming Sun ${ }^{4}$, Tong-peng Xu ${ }^{5}$, Li Yin $^{5}$, Er-bao Zhang ${ }^{4}$, Wei De ${ }^{4}$ and Yong-qian Shu ${ }^{5^{*}}$

\begin{abstract}
Background: Hepatocellular carcinoma (HCC) is one of the leading causes of cancer-related death, especially in China. And the mechanism of its progression remains poorly understood. Growing evidence indicates that long non-coding RNAs (InCRNAs) are found to be dysregulated in many cancers, including HCC. CDKN2B antisense RNA1 (ANRIL), a InCRNA, coclustered mainly with p14/ARF has been reported to be dysregulated in gastric cancer, esophageal squamous cell carcinoma, and lung cancer. However, its clinical significance and potential role in HCC is still not documented.

Methods and results: In this study, expression of ANRIL was analyzed in 77 HCC tissues and matched normal tissues by using quantitative real-time polymerase chain reaction (qRT-PCR). ANRIL expression was up-regulated in HCC tissues, and the higher expression of ANRIL was significantly correlated with tumor size and Barcelona Clinic Liver Cancer (BCLC) stage. Moreover, taking advantage of loss of function experiments in HCC cells, we found that knockdown of ANRIL expression could impair cell proliferation and invasion and induce cell apoptosis both in vitro and in vivo. We also found that ANRIL could epigenetically repress KLF2 transcription in HCC cells by binding with PRC2 and recruiting it to KLF2 promoter region. We also found that Sp1 could regulate the expression of ANRIL.
\end{abstract}

Conclusion: Our results suggest that InCRNA ANRIL, as a growth regulator, may serve as a new biomarker and target for therapy in HCC.

Keywords: Long non-coding RNA, ANRIL, HCC, Proliferation, KLF2

\section{Background}

Hepatocellular carcinoma (HCC) is the third leading cause of cancer-related death globally. Half of these deaths were estimated to occur in China [1]. The prognosis of patients with HCC remains poor despite the therapeutic advances in HCC treatment recently. Therefore, a great challenge lies ahead in the understanding of the molecular mechanisms of hepatocarcinogenesis and the identification of the new biomarkers for HCC that will supply an arm for improving diagnosis and management of human HCC.

\footnotetext{
* Correspondence: yongqian_shu@163.com

${ }^{\dagger}$ Equal contributors

${ }^{5}$ Department of Oncology, First Affiliated Hospital, Nanjing Medical University, Nanjing City, Jiangsu Province, People's Republic of China Full list of author information is available at the end of the article
}

Long non-coding RNAs (LncRNAs) are non-protein coding transcripts with a length greater than 200 nucleotides. Accumulating evidence showed that lncRNAs participated in cancer cells biological processes, such as cell growth, cell metastasis, cell differentiation, and fate decision [2-4]. Additionally, many studies demonstrate that lncRNAs play a critical role in tumorigenesis, and their misexpression confers tumor initiation and cancer cell growth and metastasis [5-7]. For example, IncRNA HOTAIR is dysregulated in many cancers $[8,9]$. Moreover, it could promote the invasion-metastasis cascade in cancer cells by binding to PRC2 [8]. In a word, there has been a heavy focus on the ways that lncRNAs contribute to cancer development. However, their aberrant 
expression and functional roles in HCC development are still not well-documented.

Among them, IncRNA CDKN2B antisense RNA 1 (ANRIL) is transcribed from the INK4b-ARF-INK4a gene cluster in the opposite direction, which has been identified as a genetic susceptibility locus shared associated by coronary disease, intracranial aneurysm, type 2 diabetes, and also cancers [10, 11]. Moreover, ANRIL could be induced by ATM-E2F1 signaling pathway and is required for the silencing of p15INK4B by recruiting PRC2 [12, 13]. In our previous study, we found that ANRIL was overexpressed and played an important role in gastric carcinogenesis and NSCLC development [14, 15]. However, the functional role and underlying mechanism of ANRIL in HCC remains unclear. Here we investigate the relationship between ANRIL and HCC. We found that ANRIL was up-regulated in HCC tissues than that in corresponding non-tumor tissues, and its up-regulation is related with tumor size and Barcelona Clinic Liver Cancer (BCLC) stage. Moreover, ANRIL could regulate cell growth both in vitro and in vivo via epigenetically silencing KLF2 by binding to PRC2. We also found that Sp1 could regulate the expression of ANRIL. Our results suggest that Sp1-induced ANRIL can regulate KLF2 expression in the epigenetic level and facilitate the development of lncRNAdirected diagnostics and therapeutics of HCC.

\section{Results}

ANRIL is up-regulated in hepatocellular carcinoma tissues and is associated with tumor size and BCLC stage

ANRIL expression was significantly up-regulated in $75.32 \%$ (58 of 77 , fold $\geqq 1.0$ ) tumor tissues compared with normal counterparts $(P<0.01)$ (Fig. 1a, b). To understand the significance of ANRIL overexpression in HCC, we investigated the potential associations between ANRIL expression and patients' clinicopathological features. Clinicopathological features of HCC patients are shown in Table 1. Noticeably, high ANRIL expression was significantly correlated with tumor size $(P<0.01)$ and advanced BCLC stage $(P<0.01)$. However, ANRIL expression was not associated with other parameters such as drinking state $(P=0.932)$, age $(P=0.850)$, gender $(P=0.608)$, AFP $(P=0.713)$, HBV $(P=0.713)$, and cirrhosis $(P=0.319)$ in HCC.

\section{ANRIL is up-regulated in HCC cell lines and could be activated by transcript factor SP1}

To investigate the functional role of ANRIL in HCC cells, quantitative real-time polymerase chain reaction (qRT-PCR) was used to detect the expression of ANRIL in three HCC cell lines. As shown in Fig. 1c, three cell lines (HepG2, HepG3B, MHCC-97H) expressed high levels of ANRIL compared with the normal hepatic epithelium cell line (L02). Previous study indicated that ANRIL expression could be activated by E2F1. In this study, we performed bioinformatics analysis and found that there are 13 SP1 binding sites in the ANRIL promoter region (as shown in Table 2), which suggest that SP1 could also regulate ANRIL transcription in HCC cells. Chromatin immunoprecipitation (ChIP) assay showed that SP1 could directly bind to ANRIL promoter regions (1081 bp) to silence ANRIL transcription. In addition, overexpression of SP1 in HCC cells could up-regulate ANRIL expression, while knockdown of SP1 in HCC cells could down-regulate ANRIL expression (as shown in Fig. 1d-k).

\section{Knockdown of ANRIL inhibits HCC cell proliferation and induces cell apoptosis in vitro}

To investigate the potential role of ANRIL on HCC cell proliferation, ANRIL siRNA was transfected into HepG2 and HepG3B cells. To ensure the efficiency of interference and avoid off-target effects, we used a validated effective interference target sequence of ANRIL, according to Kotake's study [12]. qRT-PCR assays revealed that ANRIL expression was significantly reduced after transfection with si-ANRIL (Fig. 2a). Then MTT assay showed that knockdown of ANRIL expression significantly inhibited cell proliferation both in HepG2 and HepG3B cells compared with control cells (Fig. 2b). Similarly, the result of colony formation assay revealed that clonogenic survival was significantly decreased following inhibition of ANRIL both in HepG2 and Hep3B cell lines (Fig. 2c). Next, flow cytometric analysis was performed to further examine whether the effect of ANRIL on proliferation of HCC cells by altering cell cycle progression or apoptosis. The results revealed that the cell cycle progression of HepG2/si-ANRIL and Hep3B/si-ANRIL cells was significantly stalled at the G1-G0 phase compared with cells transfected with si-NC (Fig. 2d). In addition, knockdown of ANRIL could obviously induce cell apoptosis (Fig. 2e).

\section{Effect of ANRIL on HCC cell migration and invasion}

Migration and invasion is a significant aspect of cancer progression, which involves the dissolution of extracellular matrix proteins and the migration of tumor cells into contiguous tissues. To investigate whether ANRIL had a direct functional role in cell invasion in HCC, we performed transwell assays. The results showed that inhibition of ANRIL could significantly impair HCC cells migration and invasion ability when compared with control cells (Fig. 3).

\section{ANRIL promotes HCC cell proliferation in vivo}

To further determine whether ANRIL affects tumorigenesis, we injected HepG2 cells transfected with either empty vector or sh-ANRIL into male nude mice. In consistent with in vitro results, tumor growth in the sh-ANRIL group was obviously slower than that in the empty vector group 


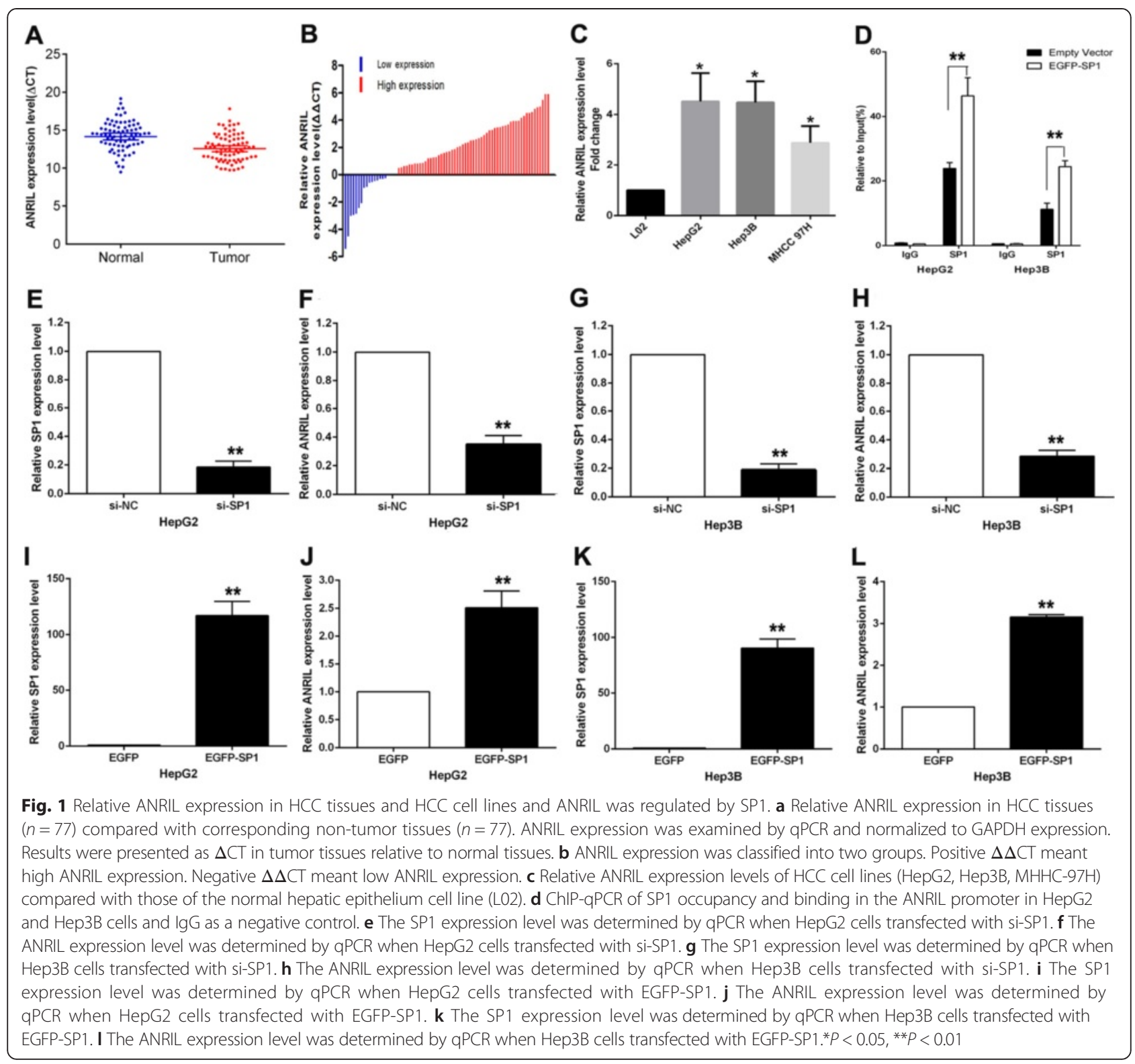

(Fig. 4a). Up to 16 days after injection, the average tumor weight in the sh-ANRIL group $(0.260 \pm 0.107 \mathrm{~g})$ was significantly lower than that in the control group $(0.442 \pm 0.716$ g) $(P<0.01)(($ Fig. 4b). qRT-PCR analysis was performed to detect the average expression of ANRIL in tumor tissues selected from mice (Fig. 4c). Results demonstrated that the average expression levels of ANRIL in the sh-ANRIL group were lower than those in the empty group. Moreover, we found that the tumors developed from empty vector transfected cells showed a stronger Ki-67 expression than that in tumors formed from shANRIL as detected by immunohistochemistry (IHC) analysis (Fig. 4d). These data further supported the role of ANRIL in HCC cell growth and proliferation.

\section{ANRIL negatively regulates expression of KLF2}

As previously reported, ANRIL could suppress p15 and p21 expression by binding with PRC2. In the present study, to investigate whether there are some other target genes that may be regulated by ANRIL, we performed coexpression analysis by using GSE45435 data from GEO datasets. The results showed that Kruppel-like factor 2 (KLF2) may be a new target of ANRIL in HCC (as shown in Fig. 5a). We also analyzed the KLF2 gene expression in HCC by using GSE 56140. It showed that KLF2 was down-regulated in HCC (as shown in Fig. 5b). And we further found that knockdown of ANRIL expression could up-regulate both KLF2 mRNA and protein expression levels in HCC cells (Fig. 5c-e). Moreover, knockdown of 
Table 1 Correlation between ANRIL expression and clinicopathological characteristics in hepatocellular carcinoma

\begin{tabular}{|c|c|c|c|}
\hline \multirow[t]{2}{*}{ Clinical parameter } & \multicolumn{2}{|l|}{ ANRIL } & \multirow{2}{*}{$\begin{array}{l}\text { Chi-squared } \\
\text { test } P \text { value }\end{array}$} \\
\hline & High no. cases & Low no. cases & \\
\hline Age (years) & & & 0.850 \\
\hline$<50$ & 23 & 8 & \\
\hline$>50$ & 35 & 11 & \\
\hline Gender & & & 0.608 \\
\hline Male & 46 & 14 & \\
\hline Female & 12 & 5 & \\
\hline Drinking state & & & 0.932 \\
\hline Yes & 36 & 12 & \\
\hline No & 22 & 7 & \\
\hline HBV & & & 0.713 \\
\hline Yes & 50 & 17 & \\
\hline No & 8 & 2 & \\
\hline Cirrhosis & & & 0.155 \\
\hline Yes & 46 & 17 & \\
\hline No & 12 & 2 & \\
\hline AFP & & & 0.625 \\
\hline$\leqq 20$ & 18 & 8 & \\
\hline 20-400 & 19 & 6 & \\
\hline$\geqq 400$ & 21 & 5 & \\
\hline Tumor size & & & $<0.01$ \\
\hline$\leqq 3 \mathrm{~cm}$ & 11 & 9 & \\
\hline $3-5 \mathrm{~cm}$ & 13 & 9 & \\
\hline $5-10 \mathrm{~cm}$ & 31 & 1 & \\
\hline$\geqq 10 \mathrm{~cm}$ & 3 & 0 & \\
\hline BCLC stage & & & $<0.01$ \\
\hline 0 & 2 & 2 & \\
\hline A & 19 & 15 & \\
\hline B & 37 & 2 & \\
\hline
\end{tabular}

EZH2 or SUZ12 could also up-regulate KLF2 mRNA and protein expression levels in HCC cells (Fig. 5f-k). We examined the ANRIL expression levels in HCC cell cytoplasm and nucleus distribution, and the results showed that ANRIL expression is more located in nucleus (seen in Fig. 5l, m). In addition, the results of RNA immunoprecipitation (RIP) assays revealed that ANRIL could directly bind with PRC2 in HCC cells (seen in Fig. 5n, o). And ChIP assays were performed to determine whether EZH2 could directly bind to KLF2 promoter regions to silence KLF2 transcription. The results showed that EZH2 can directly bind to KLF2 promoter regions (616 bp), while knockdown of ANRIL expression decreased its binding ability (seen in Fig. 5p, q). Then qRT-PCR analysis was performed to detect the average expression of KLF2 in tumor tissues from mice (Fig. 5r). Results demonstrated that the average expression levels of KLF2 in the shANRIL group were higher than those in the empty group. Finally, we found that the tumors developed from shANRIL-transfected cells showed a stronger KLF2 expression than that in tumors formed from empty vector as detected by IHC analysis (Fig. 5s). These data indicated that KLF2 was a new ANRIL target gene in HCC, and its expression can be silenced by EZH2 which is recruited by ANRIL to KLF2 promoter region and mediated H3K27 trimethylation modification.

\section{Overexpression of KLF2 impairs HCC cell proliferation and induces cell apoptosis}

To determine whether KLF2 involved in ANRIL mediated increased HCC cell proliferation, we up-regulated KLF2 expression in HCC cells by transfecting with a FLAGtagged KLF2 expression vector using the pCMV-Tag2B vector (Stratagene, Santa Clara, CA, USA). The qRT-PCR results showed that KLF2 expression is significantly upregulated in pCMV-Tag2B-KLF2-transfected HCC cells when compared with control cells (Fig. 6a). Furthermore, MTT assays and colony formation assay revealed that KLF2 overexpression inhibited HCC cell growth (Fig. 6b, c), and flow cytometric analysis indicated that increased KLF2 expression induced cell apoptosis. These data suggest that KLF2 partly involved in HCC cell proliferation and apoptosis.

\section{ANRIL negatively regulates expression of KLF2 by rescue assays}

Rescue assays were performed to determine whether ANRIL regulates HCC cell proliferation via repressing KLF2 expression. HepG2 cells were co-transfected with si-ANRIL and si-KLF2. The results of MTT and colony formation assay indicated that co-transfection could partially rescue siANRIL-impaired proliferation in HepG2 cells (Fig. 7a, b). Western blotting showed the same results (Fig. 7c).

\section{Discussion}

In recent years, the discovery of lncRNAs, which have emerged as a new and crucial layer of gene regulators, has dramatically altered our understanding of the biology of complex diseases including cancers $[16,17]$. A large number of studies have shown that dysregulated expression of lncRNAs participate in cancer progression and predict patients' outcome [18-20]. For example, GAS5 can promote the apoptosis of prostate cancer cells and its levels decline as prostate cancer cells acquire castrate-resistance, so that enhancing GAS5 expression may improve the effectiveness of chemotherapies [6]. In HCC, HULC was the first reported lncRNA that is specifically up-regulated [21, 22]. A number of lncRNAs, such as MVIH and URHC, have been reported to be involved in HCC development and 
Table 2 SP1 putative binding sites in the ANRIL promoter region by JASPAR

\begin{tabular}{|c|c|c|c|c|c|c|c|}
\hline \multicolumn{8}{|c|}{13 putative sites were predicted with these settings (90 \%) in sequence named gi 568815589: 21992791-21994791 } \\
\hline Model ID & Model name & Score & Relative score & Start & End & Strand & Predicted site sequence \\
\hline MA0079.3 & SP1 & 11.960 & 0.931611173530848 & 1241 & 1251 & 1 & ТСТССТССТСС \\
\hline MA0079.3 & SP1 & 11.933 & 0.931271482620182 & 1244 & 1254 & 1 & ССТССТССТСС \\
\hline MA0079.3 & SP1 & 11.615 & 0.927270678561222 & 1647 & 1657 & 1 & GCACCGCCCCC \\
\hline MA0079.3 & SP1 & 11.673 & 0.928000384961913 & 1664 & 1674 & 1 & TCTCCGCCCCG \\
\hline MA0079.3 & SP1 & 12.920 & 0.943689072576764 & 1702 & 1712 & 1 & CGCCCGCCCCC \\
\hline MA0079.3 & SP1 & 10.466 & 0.912814943140641 & 1709 & 1719 & 1 & CССССАCСTTC \\
\hline MA0079.3 & SP1 & 14.400 & 0.962309166939219 & 1721 & 1731 & 1 & CCCCCACCCCC \\
\hline MA0079.3 & SP1 & 11.514 & 0.925999982932433 & 1727 & 1737 & 1 & CCCCCACCCCA \\
\hline MA0079.3 & SP1 & 13.360 & 0.949224776306143 & 1732 & 1742 & 1 & ACCCCACCCCC \\
\hline MA0079.3 & SP1 & 10.179 & 0.909204154571706 & 1864 & 1874 & 1 & CTCCCGCCTAC \\
\hline MA0079.3 & SP1 & 9.496 & 0.90061123264633 & 1882 & 1892 & 1 & TTCCCGCCCTG \\
\hline MA0079.3 & SP1 & 14.400 & 0.962309166939219 & 1899 & 1909 & 1 & CCСССАССССС \\
\hline MA0079.3 & SP1 & 10.467 & 0.912827524285481 & 1919 & 1929 & 1 & ТTCССАСССТС \\
\hline
\end{tabular}

This type of analysis has a high sensitivity but abysmal selectivity. In other words, while true function will be detected in most cases, most predictions will correspond to sites bound in vitro but with no function in vivo. A number of additional constraints of the analysis can improve the prediction; phylogenetic footprinting is the most common. We recommend using the ConSite service, which uses the JASPAR datasets. The review Nat Rev Genet. 2004 Apr;5(4):276-87 gives a comprehensive overview of transcription binding site prediction [33]

progression [21, 23]. In this study, we found another lncRNA ANRIL whose expression is significantly upregulated in HCC tissues compared with normal tissues. Moreover, increased ANRIL expression was correlated with $\mathrm{HCC}$ tumor size and BCLC stage, which suggests that ANRIL may play a key role in HCC development and progression.

Recently, several studies indicated that IncRNA expression could also be regulated by some transcript factors (TF), such as c-myc which could activate HOTAIR transcription, and PVT-1 expression can be regulated by p53 [24, 25]. ANRIL expression has been reported to be regulated by a key TF E2F1 [13, 26]; however, in this study, we performed bioinformatics analysis and found that SP1 could also regulate ANRIL transcription in HCC cells. ChIP assay also showed that SP1 could directly bind to ANRIL promoter regions to silence ANRIL transcription. In addition, overexpression of SP1 in HCC cells could up-regulate ANRIL expression, while knockdown of SP1 in HCC cells could down-regulate ANRIL expression. These data showed that ANRIL expression could also be regulated by SP1 in HCC cells, which suggests that one lncRNA may be simultaneously regulated by multiple different transcript factors.

As is known, lncRNAs participated in cancer cells' biological function, and we found that knockdown of ANRIL could impair HCC cell proliferation and invasion and induce cell apoptosis both in vitro and in vivo. These data suggests that lncRNA ANRIL contributes to HCC development via regulation of cell proliferation and apoptosis. A completely different mode of action is executed by the
lncRNA ANRIL to block the activity of tumor suppressor genes. For example, ANRIL interacts with SUZ12 (a subunit of the PRC2) and recruits the complex to repress the expression of p15 (INK4B), a well-known tumor suppressor gene [13]. A similar study identified chromobox homolog 7 (CBX7), a subunit of the polycomb repressive complex 1 (PRC1) as molecular interaction partner of ANRIL, which results in the recruitment of PRC1 to the p16(INK4A)/p14(ARF) locus and silencing of this gene locus by H3K27 trimethylation [10]. However, we found that ANRIL could bind with both EZH2 and SUZ12 in HCC cells. Furthermore, bioinformatics analysis indicated that KLF2 could be a new ANRIL downstream target, and knockdown of ANRIL, EZH2 and SUZ12 expression indeed both up-regulated KLF2 expression levels in HCC cells. In addition, ChIP assays also demonstrated that EZH2 could directly bind to KLF2 promoter region and inhibition of ANRIL decreased its binding ability. Our results indicated that ANRIL could repress KLF2 transcription by binding with EZH2 and SUZ12 and recruitment of PRC2 to the KLF2 gene locus in HCC cells.

The Kruppel-like factor (KLF) family which consists of a set of transcription factors that have been identified in diverse organisms functions in cell differentiation and proliferation [27]. They have been identified as suppressors or activators of different genes in a cell type and promoter-dependent manner [28]. KLF2 is one of the critical members due to its tumor suppressor function in tumors [29, 30]. Moreover, previous study showed that EZH2 could directly bind to KLF2 promoter and silence of KLF2 expression result in blocking the tumor- 

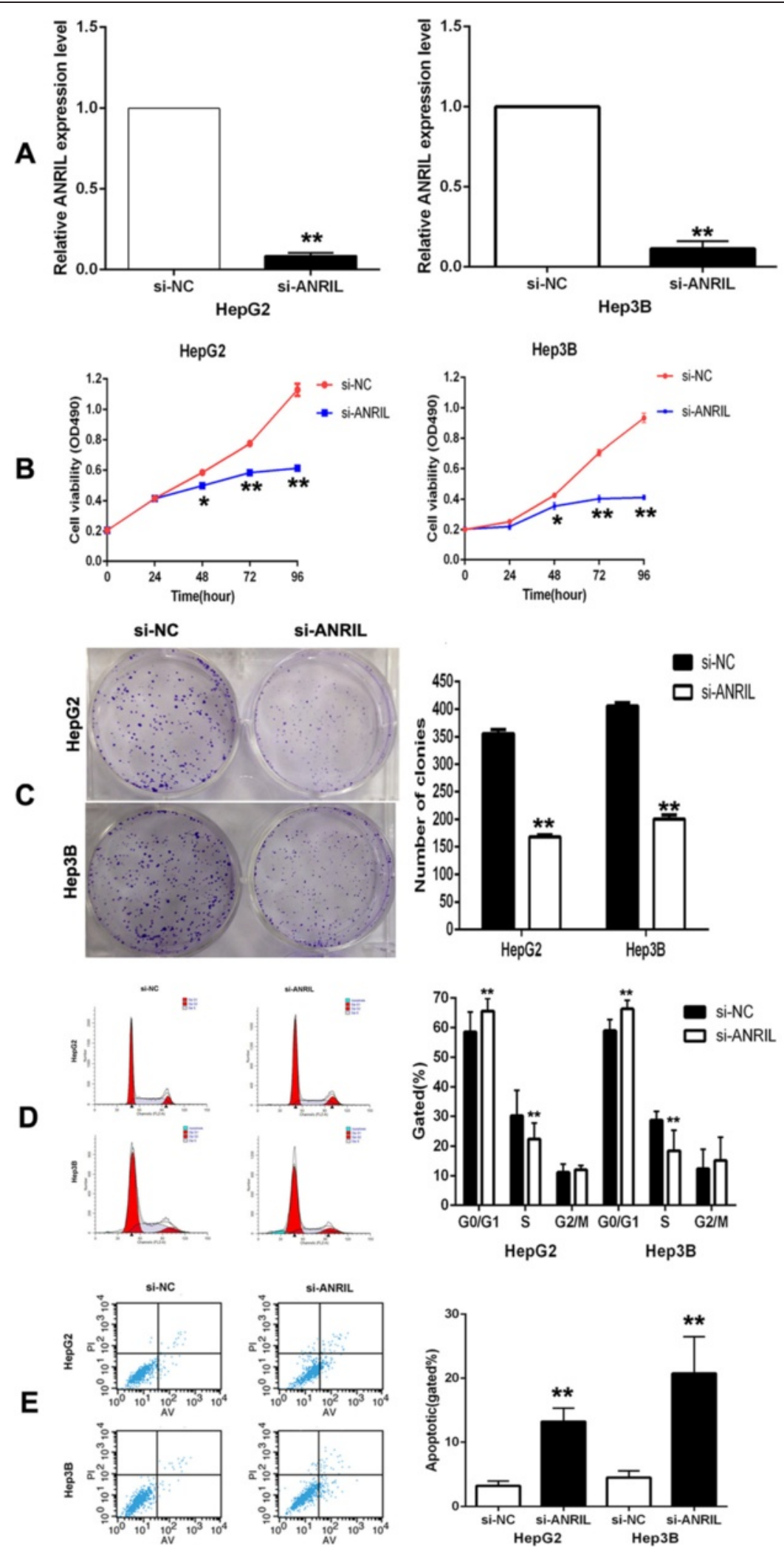

Fig. 2 (See legend on next page.) 
(See figure on previous page.)

Fig. 2 Effects of knockdown of ANRIL on HCC cell viability and apoptosis in vitro. a The ANRIL expression level was determined by qPCR when HepG2 and Hep3B cells transfected with si-ANRIL. b MTT assays were used to determine the cell viability for si-ANRIL-transfected HepG2 and Hep3B cells. Values represented the mean \pm s.d. from three independent experiments. c Colony-forming assays were conducted to determine the proliferation of si-ANRIL-transfected HepG2 and Hep3B cells. d Flow cytometry assays were performed to analyze the cell cycle progression when HCC cells transfected with si-ANRIL $24 \mathrm{~h}$ later. The bar chart represented the percentage of cells in G0/G1, S, or G2/M phase, as indicated. e Flow cytometry assays were performed to analyze the cell apoptosis when HCC cells transfected with si-ANRIL $48 \mathrm{~h}$ later. ${ }^{*} P<0.05,{ }^{* *} P<0.01$

suppressor features of KLF2, which is partly mediated by p21 [31]. Our data also showed that ANRIL could take part in HCC cell proliferation by silencing KLF2 transcription, and KLF2 overexpression further led to the decreased HCC cell proliferation and increased cell apoptosis. Furthermore, we performed rescue assays to determine whether ANRIL regulates HCC cell proliferation via repressing KLF2 expression. The results of MTT and colony formation assay indicated that co-transfection could partially rescue si-ANRIL-impaired proliferation in HepG2 cells. These data indicate that ANRIL promotes HCC cell proliferation through the down-regulation of KLF2 expression. Our results suggested that lncRNA, especially ANRIL, may influence the same cell biological function via regulating different target genes depending on different cancer cells.

\section{Conclusion}

In summary, the expression of ANRIL was significantly up-regulated in HCC tissues and cells, suggesting that its overexpression may be an important factor for HCC progression. We showed that ANRIL may regulate the proliferation ability of HCC cells partially through silencing of the KLF2 by binding with PRC2, which suggested that IncRNAs contribute to different cancer cells' biological function through regulating different genes. Further insights into the functional and clinical implications of ANRIL and its targets, which are identified as KLF2, may contribute to the understanding of HCC pathogenesis and facilitate the development of IncRNA-directed diagnostics and therapeutics against this disease.

\section{Materials and methods}

\section{Patient data and tissue samples}

A total of 77 fresh HCC tissue samples and matched normal adjacent tissue samples were selected from patients who underwent resection of HCC at Huai'an First People's Hospital, Nanjing Medical University (Huai'an, China). The HCC diagnosis was histopathologically confirmed. None of the patients received preoperative therapy. Data from all subjects were obtained from medical records, pathology reports, and personal interviews with the subjects. The collected data included gender, age, drinking state, the history of $\mathrm{HBV}$ and cirrhosis, and HCC features (e.g., tumor size, stage). HCC clinical stage was determined according to the BCLC staging classification based on the article by Bruix et al. [32]. The clinical information for all of the samples is detailed in Table 1. Fresh samples were snap-frozen in liquid nitrogen immediately after resection and stored at $-80{ }^{\circ} \mathrm{C}$. Matched non-tumor specimens were obtained from a part of the resected specimen that was farthest from the tumor.

\section{Ethical approval of the study protocol}

This study was conducted according to the principles expressed in the Declaration of Helsinki. Tissue specimen collections were made with full informed consent of all patients following institutional ethical guidelines that were reviewed and approved by Huai'an First People's Hospital, Nanjing Medical University (Huaian, China).

\section{Cell culture}

Human HCC cell lines (HepG2, Hep3B, MHCC-97H) and one normal hepatic epithelial cell line (L02, control) were provided by Dr Beicheng Sun from the Department of Hepatopancreatobiliary, First Affiliated Hospital, Nanjing Medical University (Nanjing City, Jiangsu Province, People's Republic of China). All cell lines were cultured in Dulbecco's modified Eagle's medium (DMEM) (GIBCO-BRL) medium supplemented with $10 \%$ fetal bovine serum (FBS) at $37^{\circ} \mathrm{C}$ in $5 \% \mathrm{CO} 2$.

\section{RNA extraction and qRT-PCR analysis}

The total RNA was extracted from tissues or cells with TRIzol reagent (Invitrogen, Grand Island, NY, USA), according to the manufacturer's protocol. One microgram total RNA was reverse transcribed in a final volume of $20 \mu \mathrm{L}$ under standard conditions using PrimeScript RT Reagent Kit with gDNA Eraser (Takara, Dalian, China; RR047A). After the RT reaction, $1 \mu \mathrm{L}$ of the complementary DNA was used for subsequent qRT-PCR reactions (SYBR Premix Ex Taq, TaKaRa) following the manufacturer's protocol. The results were normalized to the expression of glyceraldehyde-3-phosphate dehydrogenase (GAPDH). The qRT-PCR and data collection were carried out on ABI 7500 real-time PCR system (Applied Biosystems, Foster City, CA, USA), and results were analyzed and expressed relative to threshold cycle (CT) values, and then converted to fold changes. All primer sequences are summarized in Additional file 1: Table S1. 


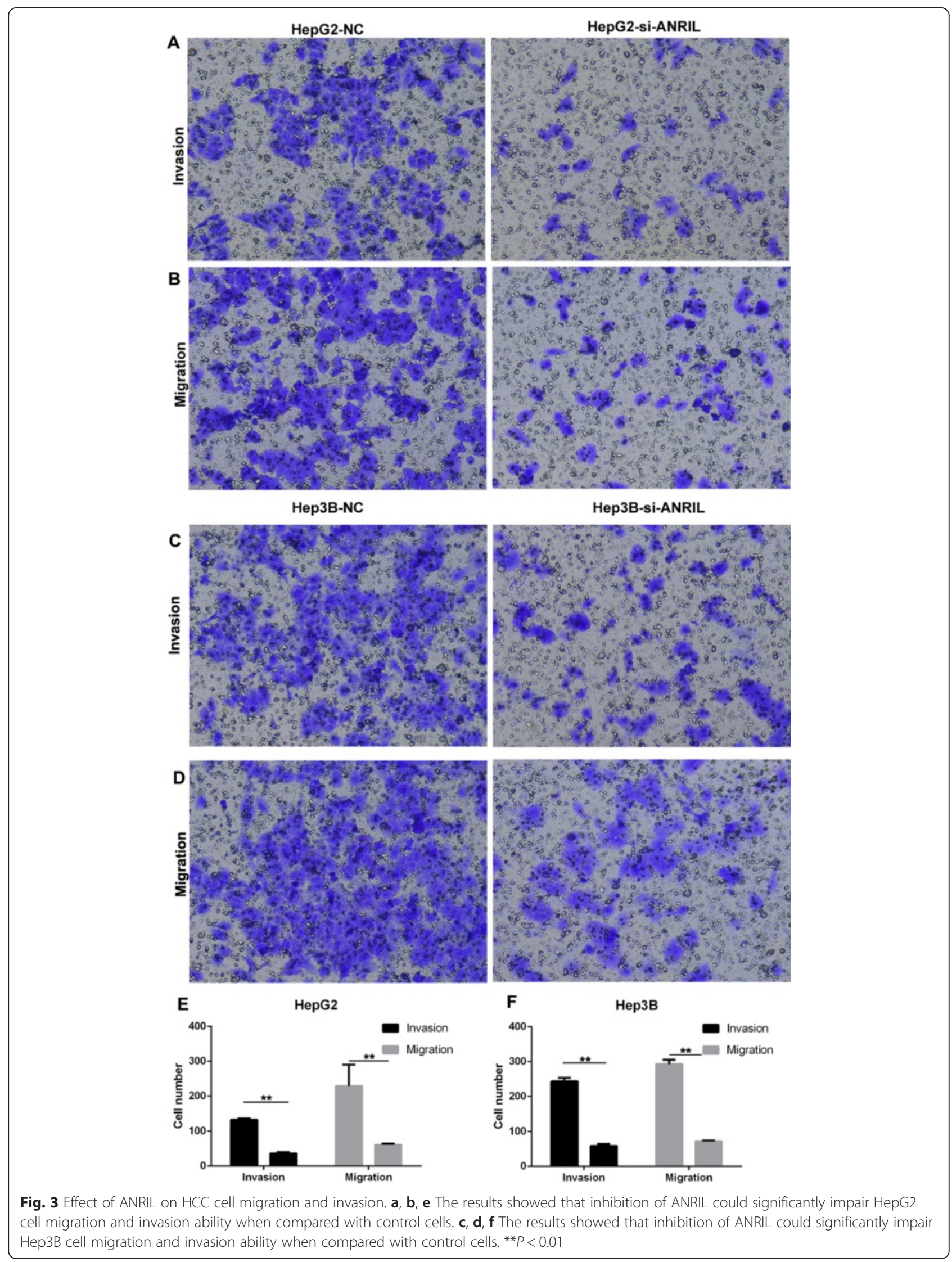




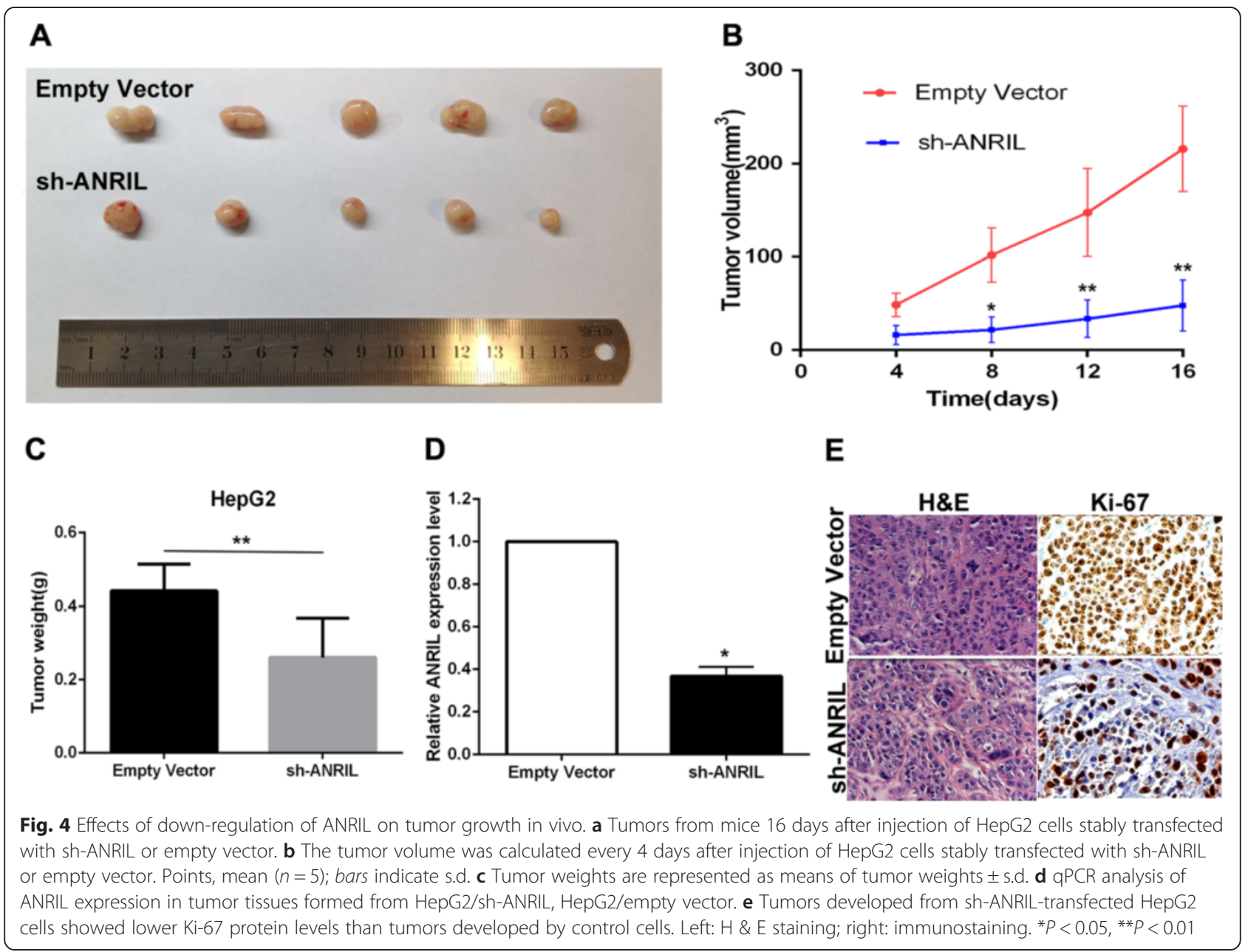

\section{Transfection of cell lines}

HCC cell lines were transfected with specific siRNA oligonucleotides. To avoid off-target effects and ensure the efficiency of interference, we used an indeed effective interference target sequence of ANRIL, according to the previous study [12]. EZH2 and SUZ12 siRNA were purchased from Realgene (Nanjing, China). Non-specific siRNA (si-NC) and si-ANRIL were purchased from Invitrogen. Typically, cells were seeded at six-well plates and then transfected the next day with specific siRNA (100 nM) and control siRNA (100 $\mathrm{nM})$ by using Lipofectamine RNAi MAX, according to the manufacturer's protocol (Invitrogen). EGFP-SP1 was purchased from Add gene. Plasmid vectors (EGFP-SP1, sh-ANRIL pCMV-Tag2BFLAG-KLF2 and empty vector) for transfection were prepared using DNA Midiprep or Midiprep kits (Qiagen, Hilden, Germany) and transfected into HepG2 and Hep3B cells.

\section{Cell proliferation assays}

Cell proliferation was monitored by Cell Proliferation Reagent Kit I (MTT) (Roche, Basel, Switzerland). The transfected cells were plated in 96-well plates (3000 cells/well). Cell proliferation was determined every $24 \mathrm{~h}$ following the manufacturer's protocol. For the colony formation assay, 500 transfected cells were placed into each well of a six-well plate and maintained in DMEM containing $10 \%$ FBS for 12 days, replacing the medium every 4 days. Colonies were fixed with methanol and stained with $0.1 \%$ crystal violet (Sigma-Aldrich, St. Louis, MO, USA) in PBS for $15 \mathrm{~min}$. The colony formation was determined by counting the number of stained colonies. Triplicate wells were measured in each treatment group.

\section{Flow cytometry for cell cycle analysis}

HepG2 or Hep3B cells for cell cycle analysis were collected $24 \mathrm{~h}$ after transfected with si-ANRIL or respective control and $48 \mathrm{~h}$ after transfected with pCMV-Tag2BKLF2 or empty vector. Then cells were stained with propidium iodide (PI) using the CycleTEST ${ }^{\mathrm{mi}}$ PLUS DNA Reagent Kit (BD Biosciences) following the protocol and analyzed by FACScan. The percentage of the cells in G0/G1, $\mathrm{S}$, and G2/M phase were counted and compared. 


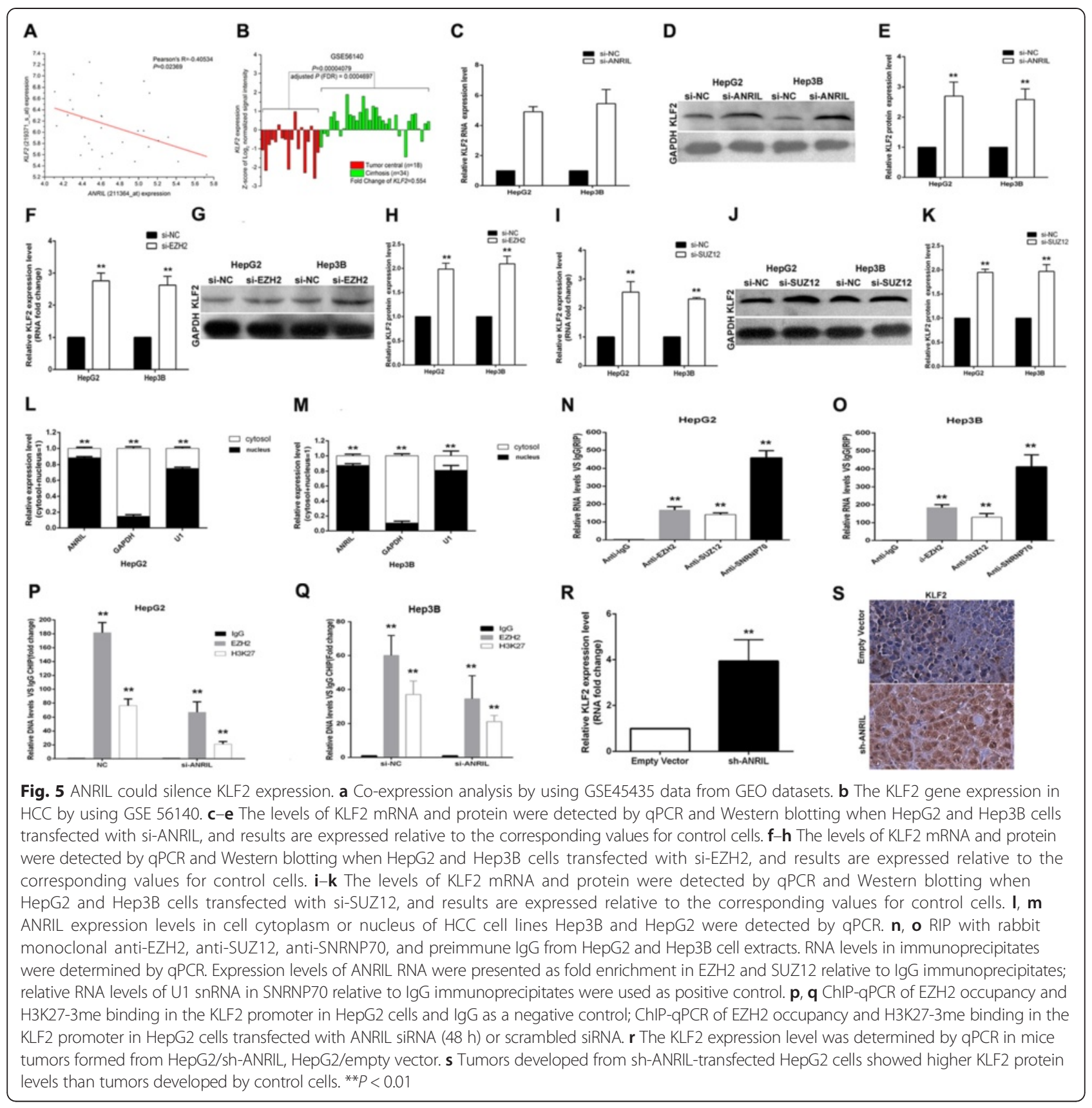

\section{Flow cytometry for cell apoptosis analysis}

HepG2 or Hep3B cells transfected with si-ANRIL, pCMVTag2B-KLF2, or respective control were harvested $48 \mathrm{~h}$ and then collected. After the double staining with FITCAnnexin V and PI was done using the FITC Annexin V Apoptosis Detection Kit (BD Biosciences) according to the manufacturer's protocol, the cells were analyzed with a flow cytometry (FACScan ${ }^{\circ}$; BD Biosciences) equipped with a CellQuest software (BD Biosciences). Cells were discriminated into viable cells, dead cells, early apoptotic cells, and apoptotic cells, and then the relative ratio of early apoptotic cells was compared to control transfectant from each experiment.

\section{Cell migration and invasion assays}

HepG2 or Hep3B cells transfected with si-ANRIL or respective control were harvested $48 \mathrm{~h}$ and then collected. For the migration assays, $5 \times 10^{4}$ cells in serum-free medium were placed into the upper chamber of an insert $(8 \mu \mathrm{m}$ pore size, Millipore). For the invasion assays, $1 \times 10^{5}$ cells in serum-free medium were placed into the upper chamber of an insert coated with Matrigel (Sigma-Aldrich). 

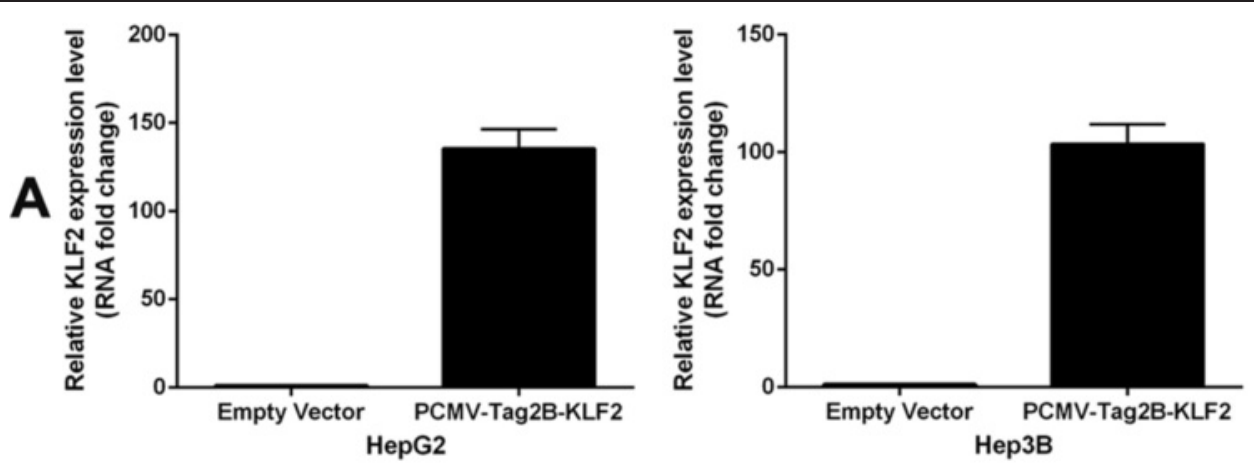

HepG2

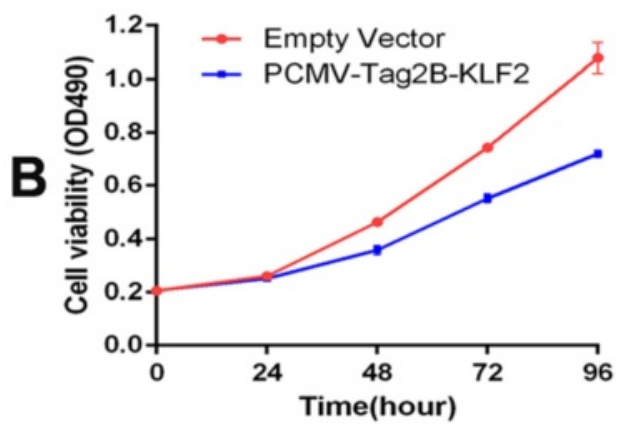

\section{Нер3B}
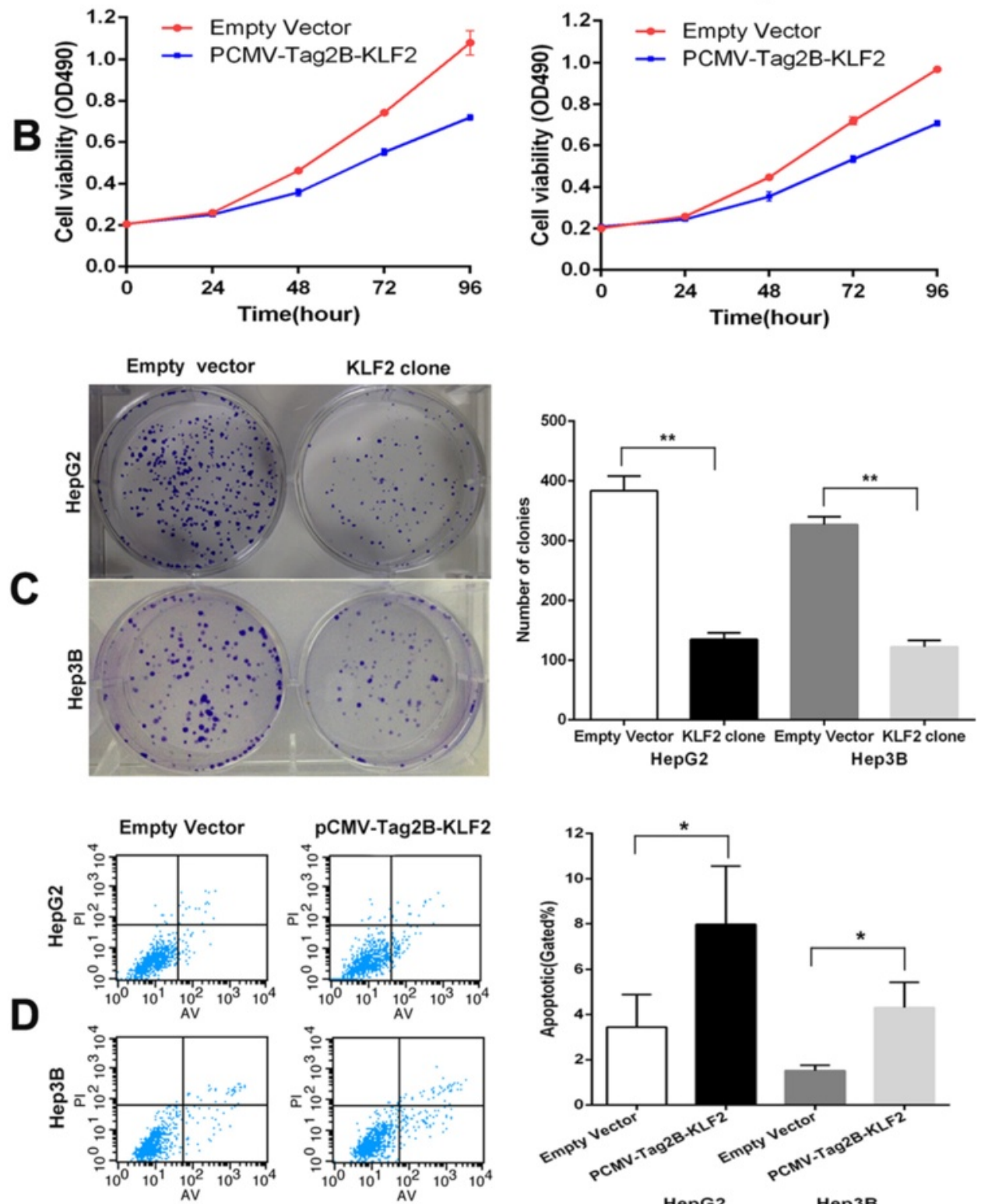

Fig. 6 (See legend on next page.)

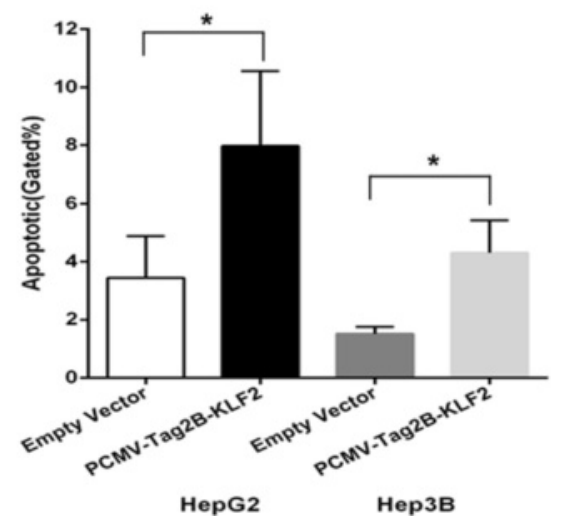


(See figure on previous page.)

Fig. 6 Overexpression of KLF2 expression inhibits HepG2 cell proliferation and improves apoptosis. a The mRNA level of KLF2 in HepG2 and Hep3B cells transfected with PCMV-Tag2B-KLF2 or empty vector was detected by qPCR. b, c MTT assays and colony-forming assays were used to determine the cell viability for PCMV-Tag2B-KLF2-transfected or empty vector-transfected HepG2 and Hep3B cells. Values represent the mean \pm s.d. from three independent experiments. $\mathbf{d}$ Apoptosis was determined by flow cytometry. UL necrotic cells, UR terminal apoptotic cells, $L R$ early apoptotic cells. ${ }^{*} P<0.05,{ }^{* *} P<0.01$

Medium containing $10 \%$ FBS was added to the lower chamber. After incubation for $24 \mathrm{~h}$, we removed the cells remaining on the upper membrane with cotton wool. Cells that had migrated or invaded through the membrane were fixed with methanol, stained with $0.1 \%$ crystal violet, imaged, and counted using an IX71 inverted microscope (Olympus, Tokyo, Japan). Experiments were repeated three times.

\section{Xenograft study}

HepG2 cells were transfected with sh-ANRIL or Scramble using Lipofectamine 2000 (Invitrogen). Forty-eight hours later, cells were collected and injected into either side of the posterior flank of the male BALB/c nude mice (4-5 weeks old). Mice were purchased from Shanghai Experimental Animal Center of the Chinese Academy of Sciences. The tumor volumes and weights were measured every 4 days in mice from the control (five mice) or sh-ANRIL (five mice) groups, and tumor volumes were calculated by using the equation $V=0.5 \times D \times d^{2}$ ( $V$, volume; $D$, longitudinal diameter; $d$, latitudinal diameter). Sixteen days after injection, the mice were killed and tumors were collected for further study (weight measure, RNA extraction, and IHC). This study was carried out strictly in accordance with the recommendations in the Guide for the Care and Use of Laboratory Animals of the National Institutes of Health. The protocol was approved by the Committee on the Ethics of Animal Experiments of Nanjing Medical University.

\section{Immunohistochemistry}

Tumors from mice were immunostained for HE, ki-67, and KLF2. The signal was amplified and visualized with 3 '-diaminobenzidine chromogen, followed by counterstaining with hematoxylin. Expression was considered to be positive when $50 \%$ or more tumor cells were stained. Anti-ki-67 (1:50) and anti-KLF2 (1:50) were purchased from R\&D company.

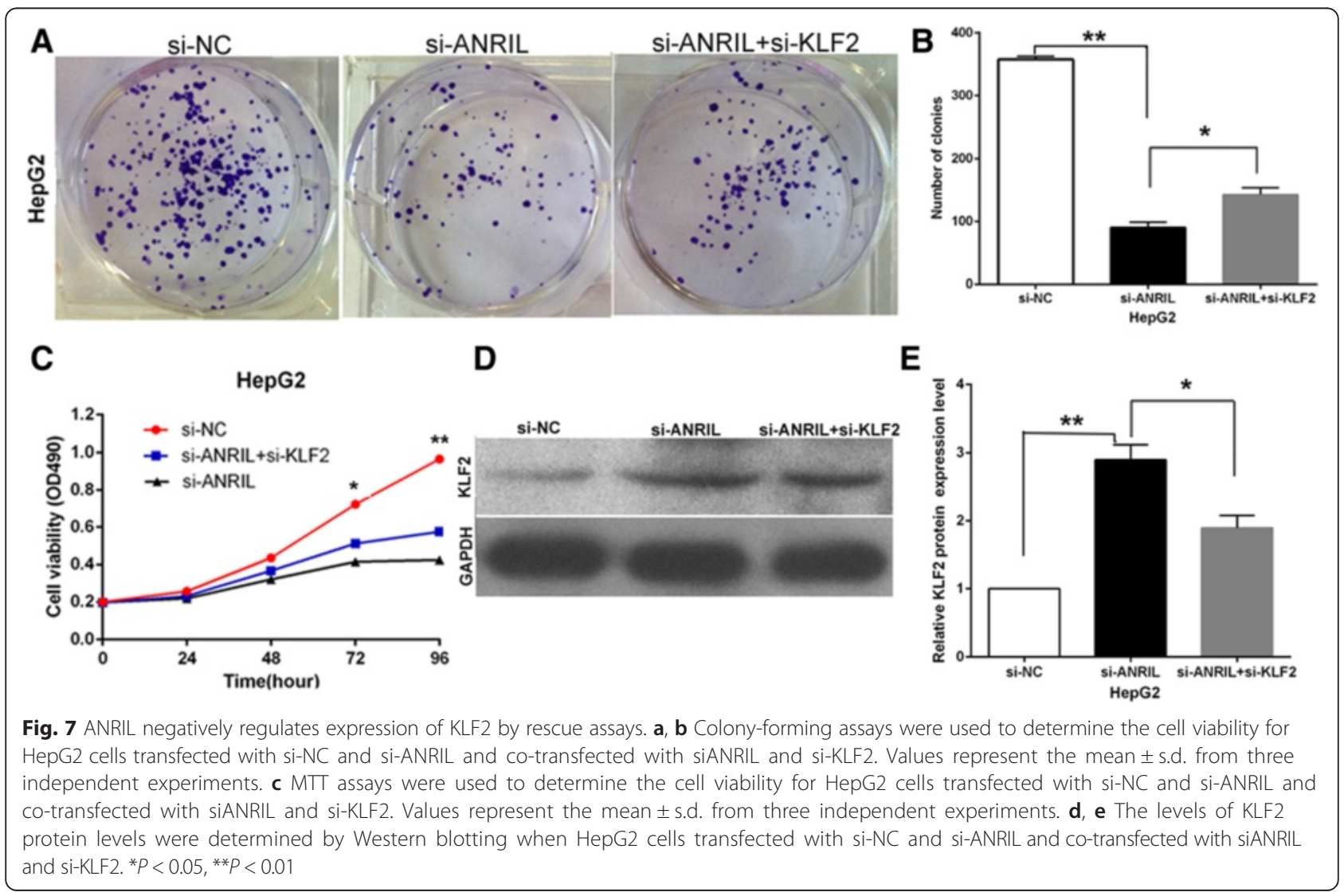




\section{Western blot assay}

Cells were lysed by using mammalian protein extraction reagent RIPA (Beyotime, Haimen, China) supplemented with protease inhibitors cocktail (Roche). Fifty micrograms of the protein extractions were separated by $10 \%$ SDS-PAGE transferred to $0.22-\mathrm{mm}$ nitrocellulose (NC) membranes (Sigma-Aldrich) and incubated with specific antibodies. The autoradiograms were quantified by densitometry (Quantity One software, Bio-Rad, Hercules, CA, USA). Anti-KLF2 was purchased from Sigma (1:1000). Results were normalized to the expression GAPDH (mouse anti-GAPDH) (Sigma (1:1000)).

\section{Subcellular fractionation location}

The separation of the nuclear and cytosolic fractions of HCC cell lines was performed according to the protocol of the PARIS Kit (Life Technologies, Carlsbad, CA, USA).

\section{Chromatin immunoprecipitation assays}

The ChIP assays were performed by using EZ-ChIP KIT according to the manufacturer's instruction (Millipore, Billerica, MA, USA). HepG2 and Hep3B cells were treated with formaldehyde and incubated for $10 \mathrm{~min}$ to generate DNA-protein cross-links. Cell lysates were then sonicated to generate chromatin fragments of 200-300 bp and immunoprecipitated with EZH2, SUZ12, and H3K27me3specific antibody (CST) or IgG as control. Precipitated chromatin DNA was recovered and analyzed by qRTPCR.

\section{RNA immunoprecipitation}

RIP experiments were performed by using a Magna RIP RNA-Binding Protein Immunoprecipitation Kit (Millipore) according to the protocol. Antibody for RIP assays of EZH2 and SUZ12 was purchased from Millipore.

\section{Statistical analysis}

All statistical analyses were performed by using SPSS 17.0 software (IBM, Chicago, IL, USA). The significance of differences between groups was estimated by the Student $t$ test, Wilcoxon test, or $X^{2}$ test. Two-sided $P$ values were calculated, and differences were considered to be statistically significant at $P<0.05$. Kendall's Tau-b and Pearson correlation analyses were used to investigate the correlation between ANRIL and KLF2 expressions.

\section{Additional file}

Additional file 1: Table S1. Summary of all primer sequences.

\section{Abbreviations}

InCRNA: Long non-coding RNA; ANRIL: CDKN2B antisense RNA1; HCC: Hepatocellular carcinoma; BCLC: Barcelona Clinic Liver Cancer; PCR: Polymerase chain reaction; RIP: RNA immunoprecipitation;
ChIP: Chromatin immunoprecipitation assays; GAPDH: Glyceraldehyde-3phosphate dehydrogenase; KLF2: Kruppel-like factor 2.

\section{Competing interests}

The authors declare that they have no competing interests.

\section{Authors' contributions}

$\mathrm{M}-\mathrm{dH}$ designed this study, detected the cells' biological function test, conducted the qRT-PCR assays, carried out the Western blotting assays, established the animal model, performed RIP and ChIP assays, done the statistical analysis, performed the immunohistochemistry assays, and drafted the manuscript. W-mC and FQ provided the tissue samples and the clinical data. RX participated in the design of the study and administrated the data analysis. MS, TX, LY, E-bZ, and WD helped to acquire the experimental data. $\mathrm{Y}$-qS conceived the study, participated in its design and coordination, and helped to draft the manuscript. All authors read and approved the final manuscript.

\section{Acknowledgements}

This study was supported by the National Natural Science Foundation of China (81172140, 81272532), Jiangsu Province Clinical Science and Technology projects (Clinical Research Center, BL2012008), and the Priority Academic Program Development of Jiangsu Higher Education Institutions (Public Health and Preventive Medicine, JX10231801). We are very grateful to Dr Beicheng Sun for providing the HCC cell lines and L02 cell line.

\section{Author details}

${ }^{1}$ Department of Medical Oncology, Huai'an First People's Hospital, Nanjing Medical University, Huai'an City, Jiangsu Province 223301, People's Republic of China. '2Department of Oncology, Jining No.1 People's Hospital, No.6, Jiankang Road, Jining City, Shandong Province 272011, People's Republic of China. ${ }^{3}$ Department of Hepatopancreatobiliary Surgery, Huai'an First People's Hospital, Nanjing Medical University, Huai'an City, Jiangsu Province 223300, People's Republic of China. ${ }^{4}$ Department of Biochemistry and Molecular Biology, Nanjing Medical University, Nanjing City, Jiangsu Province, People's Republic of China. ${ }^{5}$ Department of Oncology, First Affiliated Hospital, Nanjing Medical University, Nanjing City, Jiangsu Province, People's Republic of China.

Received: 16 January 2015 Accepted: 26 March 2015

Published online: 29 May 2015

\section{References}

1. Torre LA, Bray F, Siegel RL, Ferlay J, Lortet-Tieulent J, Jemal A. Global cancer statistics, 2012. CA Cancer J Clin. 2015.

2. Ulitsky I, Bartel DP. LincRNAs: genomics, evolution, and mechanisms. Cell. 2013;154:26-46.

3. Batista PJ, Chang HY. Long noncoding RNAs: cellular address codes in development and disease. Cell. 2013;152:1298-307.

4. Geisler S, Coller J. RNA in unexpected places: long non-coding RNA functions in diverse cellular contexts. Nat Rev Mol Cell Biol. 2013;14:699-712.

5. Chakravarty D, Sboner A, Nair SS, Giannopoulou E, Li R, Hennig S, et al. The oestrogen receptor alpha-regulated InCRNA NEAT1 is a critical modulator of prostate cancer. Nat Commun. 2014;5:5383.

6. Yacqub-Usman K, Pickard MR, Williams GT. Reciprocal regulation of GAS5 IncRNA levels and mTOR inhibitor action in prostate cancer cells. Prostate 2015.

7. Xu TP, Huang MD, Xia R, Liu XX, Sun M, Yin L, et al. Decreased expression of the long non-coding RNA FENDRR is associated with poor prognosis in gastric cancer and FENDRR regulates gastric cancer cell metastasis by affecting fibronectin1 expression. J Hematol Oncol. 2014;7:63.

8. Gupta RA, Shah N, Wang KC, Kim J, Horlings HM, Wong DJ, et al. Long non-coding RNA HOTAIR reprograms chromatin state to promote cancer metastasis. Nature. 2010;464:1071-6.

9. Kogo R, Shimamura T, Mimori K, Kawahara K, Imoto S, Sudo T, et al. Long noncoding RNA HOTAIR regulates polycomb-dependent chromatin modification and is associated with poor prognosis in colorectal cancers. Cancer Res. 2011;71:6320-6.

10. Yap KL, Li S, Munoz-Cabello AM, Raguz S, Zeng L, Mujtaba S. Molecular interplay of the noncoding RNA ANRIL and methylated histone H3 lysine 27 by polycomb CBX7 in transcriptional silencing of INK4a. Mol Cell. 2010;38:662-74. 
11. Pasmant $E$, Laurendeau I, Heron D, Vidaud M, Vidaud D, Bieche I. Characterization of a germ-line deletion, including the entire INK4/ARF locus, in a melanoma-neural system tumor family: identification of ANRIL, an antisense noncoding RNA whose expression coclusters with ARF. Cancer Res. 2007;67:3963-9.

12. Kotake Y, Nakagawa T, Kitagawa K, Suzuki S, Liu N, Kitagawa M, et al. Long non-coding RNA ANRIL is required for the PRC2 recruitment to and silencing of p15 (INK4B) tumor suppressor gene. Oncogene. 2011;30:1956-62

13. Wan G, Mathur R, Hu X, Liu Y, Zhang X, Peng G, et al. Long non-coding RNA ANRIL (CDKN2B-AS) is induced by the ATM-E2F1 signaling pathway. Cell Signal. 2013;25:1086-95.

14. Zhang EB, Kong R, Yin DD, You LH, Sun M, Han L, et al. Long noncoding RNA ANRIL indicates a poor prognosis of gastric cancer and promotes tumor growth by epigenetically silencing of miR-99a/miR-449a. Oncotarget. 2014;5:2276-92.

15. Nie FQ, Sun M, Yang JS, Xie M, Xu TP, Xia R, et al. Long noncoding RNA ANRIL promotes non-small cell lung cancer cell proliferation and inhibits apoptosis by silencing KLF2 and P21 expression. Mol Cancer Ther. 2015;14:268-77.

16. Ponting $C P$, Oliver $P L$, Reik W. Evolution and functions of long noncoding RNAs. Cell. 2009;136:629-41.

17. Tsai MC, Manor O, Wan Y, Mosammaparast N, Wang JK, Lan F, et al. Long noncoding RNA as modular scaffold of histone modification complexes. Science. 2010;329:689-93.

18. Wilusz JE, Sunwoo H, Spector DL. Long noncoding RNAs: functional surprises from the RNA world. Genes Dev. 2009;23:1494-504.

19. Sun M, Liu XH, Wang KM, Nie FQ, Kong R, Yang JS, et al. Downregulation of BRAF activated non-coding RNA is associated with poor prognosis for non-small cell lung cancer and promotes metastasis by affecting epithelial-mesenchymal transition. Mol Cancer. 2014;13:68.

20. Hirata H, Hinoda Y, Shahryari V, Deng G, Nakajima K, Tabatabai ZL, et al. Long noncoding RNA MALAT1 promotes aggressive renal cell carcinoma through Ezh2 and interacts with miR-205. Cancer Res 2015.

21. Cui M, Xiao Z, Wang $Y$, Zheng $M$, Song T, Cai $X$, et al. Long noncoding RNA HULC modulates abnormal lipid metabolism in hepatoma cells through an miR-9-mediated RXRA signaling pathway. Cancer Res. 2015;75:846-57.

22. Panzitt K, Tschernatsch MM, Guelly C, Moustafa T, Stradner M, Strohmaier HM, et al. Characterization of HULC, a novel gene with striking up-regulation in hepatocellular carcinoma, as noncoding RNA. Gastroenterology. 2007;132:330-42.

23. Xu WH, Zhang JB, Dang Z, Li X, Zhou T, Liu J, et al. Long non-coding RNA $\mathrm{URHC}$ regulates cell proliferation and apoptosis via ZAK through the ERK/MAPK signaling pathway in hepatocellular carcinoma. Int J Biol Sci. 2014;10:664-76

24. Ma MZ, Li CX, Zhang Y, Weng MZ, Zhang MD, Qin YY, et al. Long non-coding RNA HOTAIR, a c-Myc activated driver of malignancy, negatively regulates miRNA-130a in gallbladder cancer. Mol Cancer. 2014;13:156.

25. Barsotti AM, Beckerman R, Laptenko O, Huppi K, Caplen NJ, Prives C. p53-Dependent induction of PVT1 and miR-1204. J Biol Chem. 2012;287:2509-19.

26. Sato K, Nakagawa H, Tajima A, Yoshida K, Inoue I. ANRIL is implicated in the regulation of nucleus and potential transcriptional target of E2F1. Oncol Rep. 2010;24:701-7.

27. Black AR, Black JD, Azizkhan-Clifford J. Sp1 and Kruppel-like factor family of transcription factors in cell growth regulation and cancer. J Cell Physiol. 2001;188:143-60

28. Kaczynski J, Cook T, Urrutia R. Sp1- and Kruppel-like transcription factors. Genome Biol. 2003;4:206.

29. Ebert R, Zeck S, Meissner-Weigl J, Klotz B, Rachner TD, Benad P, et al. Kruppel-like factors KLF2 and 6 and Ki-67 are direct targets of zoledronic acid in MCF-7 cells. Bone. 2012;50:723-32.

30. Yamada T, Park CS, Mamonkin M, Lacorazza HD. Transcription factor ELF4 controls the proliferation and homing of CD8+ T cells via the Kruppel-like factors KLF4 and KLF2. Nat Immunol. 2009:10:618-26.

31. Wong JC, Guo L, Peng Z, Zhang W, Zhang N, Lai W, et al. Application of p21 and klf2 reporter gene assays to identify selective histone deacetylase inhibitors for cancer therapy. Bioorg Med Chem Lett. 2011;21:110-6.

32. Llovet JM, Bru C, Bruix J. Prognosis of hepatocellular carcinoma: the BCLC staging classification. Semin Liver Dis. 1999;19:329-38.

33. Wasserman WW, Sandelin A. Applied bioinformatics for the identification of regulatory elements. Nat Rev Genet. 2004;5:276.

\section{Submit your next manuscript to BioMed Central and take full advantage of:}

- Convenient online submission

- Thorough peer review

- No space constraints or color figure charges

- Immediate publication on acceptance

- Inclusion in PubMed, CAS, Scopus and Google Scholar

- Research which is freely available for redistribution

Submit your manuscript at www.biomedcentral.com/submit 